\title{
Precise temperature calibration for laser heat treatment
}

\author{
Marko Seifert ${ }^{1}$, Klaus Anhalt ${ }^{2}$, Christoph Baltruschat ${ }^{2}$, Steffen Bonss ${ }^{1}$, Berndt Brenner $^{1}$ \\ ${ }^{1}$ Fraunhofer IWS Dresden, Winterbergstrasse 28, 01277 Dresden, Germany \\ ${ }^{2}$ Physikalisch- Technische Bundesanstalt, Abbestr. 2-12, 10587 Berlin, Germany \\ E-Mail: marko.seifert@iws.fraunhofer.de
}

\begin{abstract}
:
A new induction heated fixed-point device for calibration of temperature measurement devices, typically used in laser heat treatment for the temperature range between $1000{ }^{\circ} \mathrm{C}$ and $1500{ }^{\circ} \mathrm{C}$, was developed. To define the requirements for the calibration method, selected measurement set-ups were compared and process data and results of industrial processes were analyzed. Computer simulation with FEM and FDM was used to optimize the system components and processing parameters of the induction heating of fixed-point cells. The prototype of the fixed-point device was tested successfully and first measuring results are shown. The new calibration method is expected to improve the quality and reproducibility of industrial heat treatment processes with temperature control.
\end{abstract}

Key words: fixed-point device, calibration, laser heat treatment, temperature control

\section{Introduction}

The technology of laser surface heat treatment for steel or cast iron parts with high power diode lasers was developed in the late 1990's and has been established in industrial mass production for more than 10 years (see e.g. [1]). Because of the high power density of the laser heat sources and the high processing temperatures often desired to be close to the melting temperature of the materials a precise temperature measurement and control is essential to hold the process stable and to ensure a reproducible quality of the parts. For a successful heat treatment of special high alloyed steel grades or gray cast iron surfaces the process temperature needs to be controlled within a band of only a few Kelvin. Temperatures too high can cause heavy melting of the parts surface and temperatures too low result in lower surface hardness and smaller heat penetration depths.

For the temperature measurement radiation thermometers and thermal imaging systems are used. One key problem is the non-linear signal damping by the laser optics in the case of coaxial view of the measurement device. Very critical is the pollution and wear of optical components during their lifetime, i.e. caused by processing fumes, and the connected drift of the measuring signal damping.

Therefore a fast and precise calibration method is required. Conventional blackbody or fixedpoint devices are mostly too large and too slow for this task, not flexible enough and hardly transportable. On-site calibration is needed because the temperature measurement devices are mechanically and electrically integrated into very complex and large machine systems.

To close the gap between existing calibration methods ([2], [3]) and the demands from the industry a mobile induction heated fixed-point device for fast calibration in the temperature range from 1000 to $1500{ }^{\circ} \mathrm{C}$ was developed in the content of the EMRP project HiTeMS [4]. Different variants of temperature measurement configurations, typically used in industrial laser heat treatment, were investigated to define the requirements for the device. The geometry and arrangement of system components and the processing parameters of the induction heated fixed-point device were optimized by a computer simulation with FEM and FDM.

Based on the results a prototype of an induction heated fixed-point device was developed and tested.

\section{Temperature measurement in laser heat treatment}

The temperature measurement device can be used lateral or co-axial to the laser optics (fig. $1)$. In the case that the pyrometer is mounted lateral to the laser optics it is looking directly to the laser interaction zone at the parts surface under an angle to the laser beam axis. The measuring spot of the pyrometer has to be adjusted to the position of maximum temperature very carefully. The measuring 
signal can get lost, if part edges shadow the pyrometer measuring path, i.e. if heat treating the ground of a groove or a bore hole. Even though this variant is used for industrial mass production, the efforts to ensure a reproducible quality are high.

Main advantage of the direct measurement is the fact that the measurement is not influenced by the laser optics (i.e. by signal damping) and the calibration values of the manufacturer can be used. Nevertheless contamination of the pyrometer optics can cause significant measuring errors (compare tab. 1) and calibration and adjustment is needed regularly.

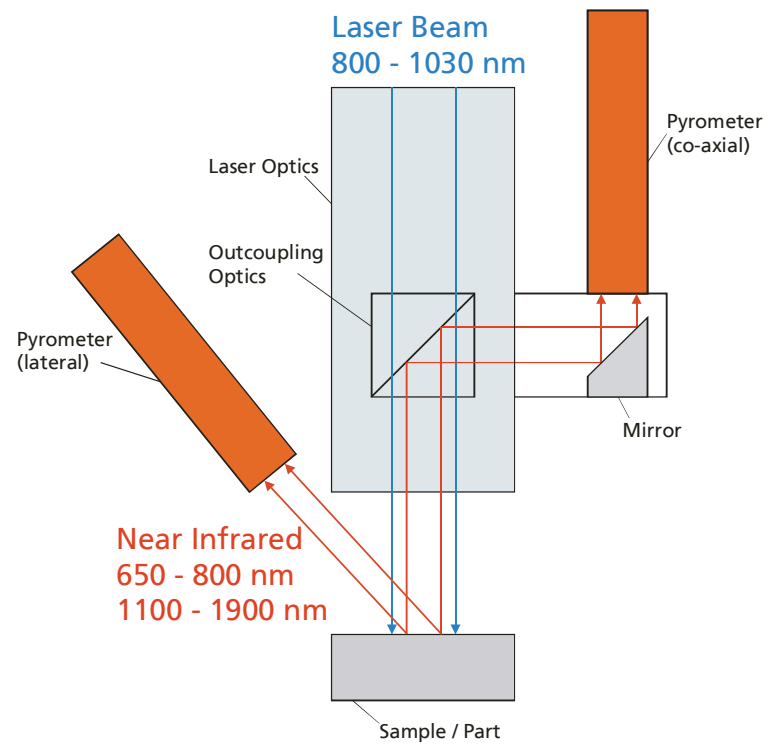

Fig. 1: Schematic diagram - variants of assembling a temperature measurement device for laser heat treatment

Because of the known problems with lateral pyrometer measurement a co-axial measurement through the laser optics is preferably used today. For this task special optical components are used, which let the laser radiation pass and reflect the NIR measuring wavelength towards the pyrometer (fig. 1). If this outcoupling plate is at the position of the collimated laser beam, the pyrometer is focused by the laser lenses, shape and size of the measuring spot are changed. Using a pyrometer co-axial at the laser optics requires a correction of the characteristic curve of the pyrometer or even a fully new calibration in the mounted state. An example of co-axial temperature measurement in industrial mass production is shown in figure 2 .

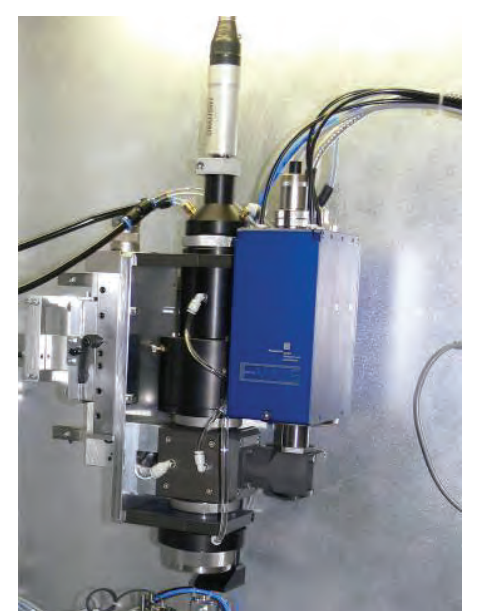

Fig. 2: Thermal imaging system E-MAqS mounted at special ring optics for co-axial temperature measurement in industrial mass production of valves

The investigation of typical measurement setups for laser heat treatment has shown that the best wavelength range for temperature measurement between 1000 and $1500{ }^{\circ} \mathrm{C}$ is $650 \mathrm{~nm}$ to $2100 \ldots 2500 \mathrm{~nm}$. The wavelength band of the laser heat sources $(800-1070 \mathrm{~nm})$ is not available for a temperature measurement, because the laser radiation overlaps the NIR temperature radiation of the glowing object. To avoid measurement errors special blocking filters with a high optical density at the discrete laser wavelength or wavelength band have to be used.

Towards longer wavelengths the working wavelength of the pyrometer is limited by the optical properties of laser lenses, additional beam shaping optics, shielding glasses and outcoupling optics typically made of fused silica and optimized with special coatings.

Because the interaction time of the laser during a full heat treatment process is within the range of milliseconds to seconds a short response time of the measuring sensor is required.

If all the preconditions for temperature measurement in laser heat treatment are taken into consideration, thermal radiation detectors based on the widely-used silicon or InGaAs photo diodes are suited for laser heat treatment.

\section{Requirements for accuracy of the temperature measurement}

The requirements for the accuracy of the measurement devices are given by the dependency of the heat treatment result on the temperature. As result of a typical industrial process the hardness distribution across the hardening zone is measured and surface hardness and hardening are depth detected. 


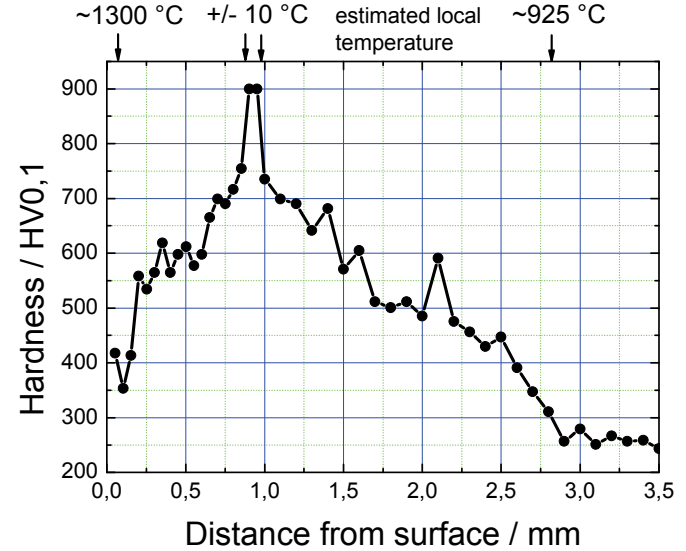

Fig. 3: Hardness in dependence of the distance from the laser heat treated surface, pre-heated part made of $X 155 \mathrm{CrVMo12.1}$, temperature gradient estimated from experimental data of heat treated samples

Figure 3 shows an example with a strong dependence of the local hardness on the maximum local temperature during the hardening process. The hardness-temperaturecurve shows a maximum at a temperature around $1200{ }^{\circ} \mathrm{C}$. The decrease of the hardness towards higher temperatures is caused by stabilization of residual austenite that is not transformed to martensite after cooling down to room temperature. To generate a high hardness level $>700 \mathrm{HV}$ the absolute surface temperature has to be within a band of several 10 Kelvin.

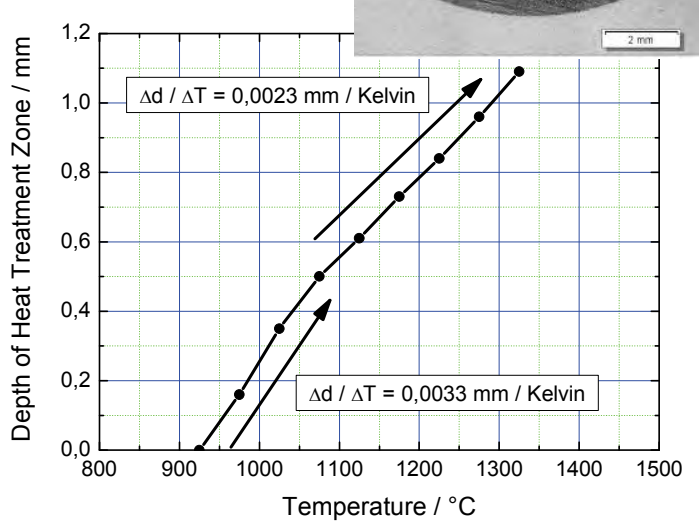

Fig. 4: Depth of the hardening zone (d) in dependence of the surface temperature $(T)$, flat samples made of tool steel X155CrMoV12.1, depth was measured in the etched cross section (example at top of diagram)

The dependency of the process temperature on the hardening result was analyzed for samples of carbon and tool steel and was investigated for selected industrial applications. Figure 4 shows one example.

If the surface temperature exceeds the material specific austenite start temperature during the heat treatment process $\left(\sim 900{ }^{\circ} \mathrm{C}\right.$ in fig. 4) the hardening process begins and after cooling down a hardening depth is generated. At surface temperatures above this threshold the hardening depth shows a strong dependence on the maximum surface temperature. For typical laser heat treatment processes with processing speed of $100-500 \mathrm{~mm} / \mathrm{min}$ the hardening depth is changed by several $0.001 \mathrm{~mm} /$ Kelvin.

Not only the generation of a reproducible hardening result but also good process stability is an important criterion for a reliable industrial production.

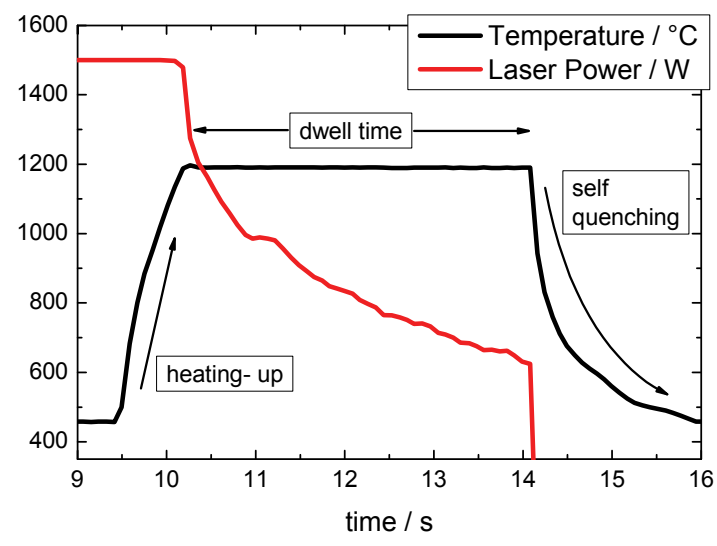

Fig. 5: Process data of a temperature controlled laser heat treatment process of the end face of a screw, temperature measured with pyrometer MAURER KTR 1075

To ensure the optimum surface temperature during the process precise temperature controllers are used. In dependence of the material, geometry and the surface quality of the parts temperature fluctuations of \pm 1 to \pm 10 Kelvin are typical for industrial processes today (fig. 5).

In particular cases a temperature deviation of +5 to +10 Kelvin can cause instable laser processes with oscillating temperature because of localized accelerated growth, delamination and the resulting overheating of oxide layers in combination with overreaction of the temperature controller.

In conclusion an accuracy and reproducibility of temperature measurement in laser heat treatment down to 5 Kelvin and a calibration method with an uncertainty significantly lower are required.

\section{Calibration and inspection of temperature measurement devices}

The calibration of radiation thermometers with blackbody or fixed-point radiators is established at national metrology institutes [2]. Selected temperature measurement devices, typically used in laser heat treatment, were tested and analyzed at the PTB Berlin using the variable temperature blackbody HTBB3200pg. 
Different influencing factors on the indicated temperature value were investigated, e.g. contamination of the optical components, a varying working distance, defocusing of the optics and varying the aperture size of the blackbody emitter. As one result it could be proved that temperature measurement devices, typically used in laser heat treatment, have the potential to measure temperatures with accuracy down to a few Kelvin. But a variety of influencing factors can cause total measuring errors in a dimension of several 10 Kelvin.

Tab. 1 illustrates the effect of contaminated pyrometer optics and Fig. 6 depicts the effect of varying the furnace aperture between $6 \mathrm{~mm}$ and $30 \mathrm{~mm}$, the so called size-of-source-effect.

Tab. 1: Influence of contamination of pyrometer optics for temperature measurement with pyrometer MAURER KTR 1075 (manufacturer calibration) at PTB Berlin. The contaminated front lense has been used for several years for laser processing in a laterally assembled pyrometer viewing unprotected at the laser/metal interaction zone.

\begin{tabular}{|c|c|}
\hline $\mathrm{T}_{\text {Blackbody }} /{ }^{\circ} \mathrm{C}$ & $\mathrm{T}_{\text {Clean }}-\mathrm{T}_{\text {Contaminated }} / \mathrm{K}$ \\
\hline 1004 & 18 \\
\hline 1113 & 18 \\
\hline 1222 & 19 \\
\hline 1300 & 26 \\
\hline 1409 & 32 \\
\hline
\end{tabular}

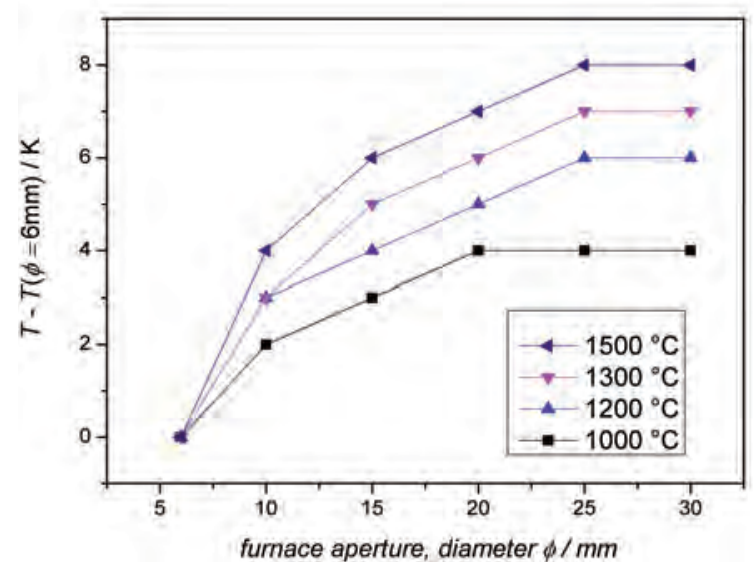

Fig. 6: Influence of the size-of-source effect (SSE) on the temperature characteristics of a thermal imaging system based on a CCD camera

To achieve the aimed accuracy with temperature measurement devices that are electrically, optically and mechanically fully integrated into complex machines at industrial sites, the on-site calibration and inspection with mobile devices is needed. Only the in situ calibration in the final machine set-up guarantees a high precision of the temperature measurement. For this reason a portable fixed- point calibration device, based on metal-carbon eutectic high-temperature fixed points, was developed for the use in temperature range between $1084.6{ }^{\circ} \mathrm{C}$ and $1492{ }^{\circ} \mathrm{C}$. In order to achieve a compact design and fast operability the fixed point is inductively heated. The design and operational characteristics of this calibration device were investigated using FEM and FDM simulation.

\section{Results of FEM and FDM simulation}

Induction heating is an established technology to heat up electrically conductive materials to high temperatures and expected to be suited for fixed-points.

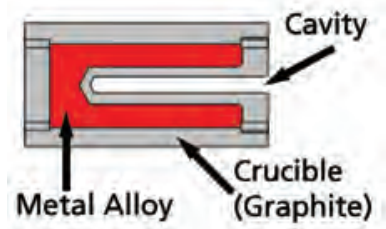

Fig. 7: Cross section of a typical fixed-point cell (design by PTB Berlin), cavity diameter $3 \mathrm{~mm}$, outer crucible diameter $25 \mathrm{~mm}$, length $40 \mathrm{~mm}$

Figure 7 shows a typical geometry of a fixedpoint cell used in calibration furnaces. Aim of the theoretical optimization of the induction coil design and the composition and dimension of components was to achieve a good homogeneity of the temperature field along the furnace axis. This is essential to get a homogeneous melting of the metal alloy and a sharp and precise inflection point in the melt or freeze temperature curves (fig. 13, 14).

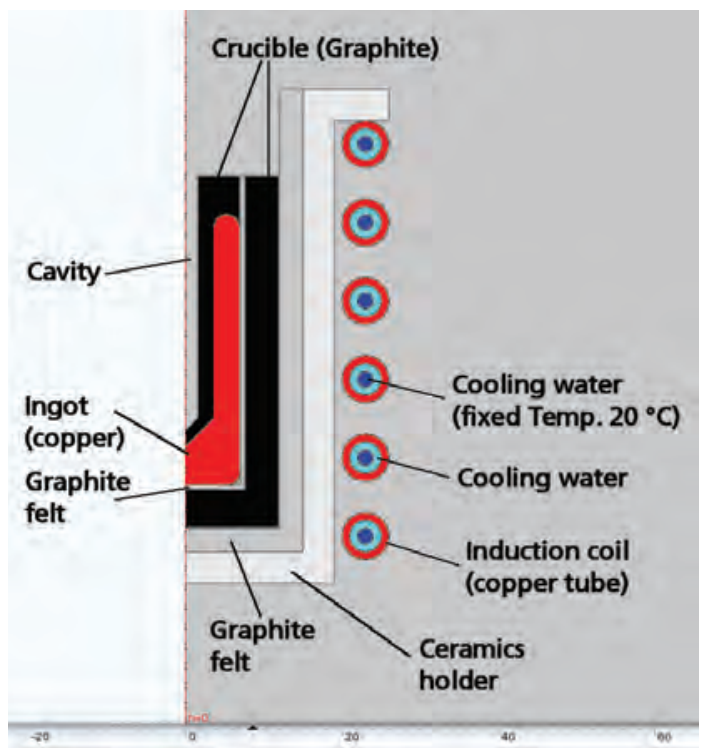

Fig. 8: Components of computer model for induction heating of fixed-point cells

Figure 8 shows the main components of the FEM computer model. Graphite felt is used for thermal insulation of the inner 
components that are heated up to $1500{ }^{\circ} \mathrm{C}$. A special ceramics holder is used for electrical insulation of the graphite components and the induction coil and for additional thermal insulation. This composition is expected to have the best efficiency and a good thermal long-term stability.

As one important result of the FEM simulation a strong influence of the induction frequency on the temperature field was found. The high penetration depth of the electromagnetic fields at low frequencies causes a significant direct heating of the metal alloy and high temperature gradients across the fixed-point cell.

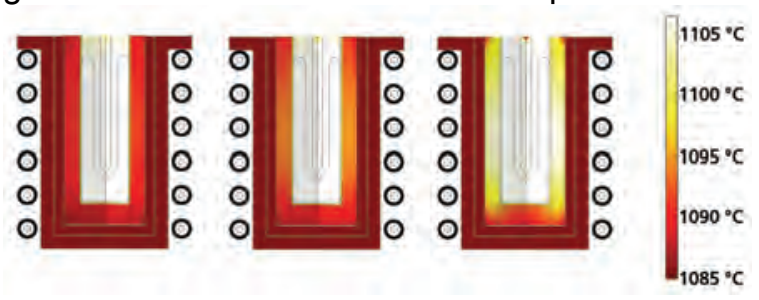

Fig. 9: FEM simulation of temperature field across a fixed-point cell after induction heating (variation of induction frequency: left: $125 \mathrm{kHz}$, middle: $250 \mathrm{kHz}$, right: $1000 \mathrm{kHz}$ )

A FDM model was developed to analyze the influence of the distribution of the induction heat sources and the resulting temperature fields on the melting process of the metal alloy.

The simulation results show that the cavity temperature is stabilized by the melting metal alloy even in the case of inhomogeneous induction heating. But the temperature gradient across the cavity wall is strongly influenced by the distribution of the inner heat sources. The inhomogeneous melting of the alloy causes a distortion of the temperature curve around the melting temperature and a slight shift of the inflection point, depending on the field of view of the pyrometer or thermal imaging system.

If the location of the inner induction heat sources is varied, the run-out of the temperature curve after the melting plateau changes drastically (fig. 10). The following general relationship was found: If the density of the induction heat sources is higher at the bottom of the cell the melting plateau is short and the temperature curve has a steep run-out. In the opposite, a high induction power density at the top of the cell causes a long plateau and long and soft run-out of the temperature curve. The knowledge of this behavior can be used for adjustment of the position of the fixed-point cell relative to the induction coil.

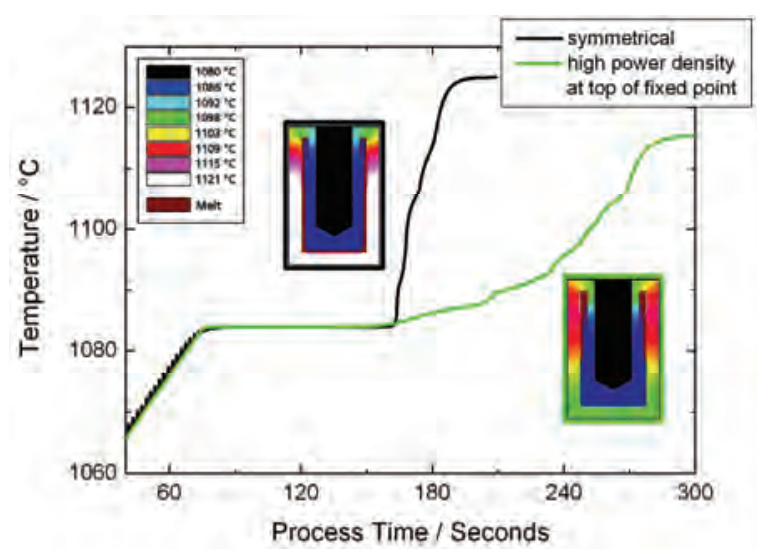

Fig. 10: Dependence of cavity temperature of copper fixed-point cell on distribution of the induction heat sources, result of FDM simulation, thermal images show the temperature field during the melting plateau

\section{Design and realization of the fixed-point device}

The heat source for fixed-point heating is an induction coil with 6 loops, working at a frequency up to $420 \mathrm{kHz}$. The fixed-point cell itself is insulated with graphite felt and placed in a ceramics crucible to avoid a direct electrical contact to the induction coil.

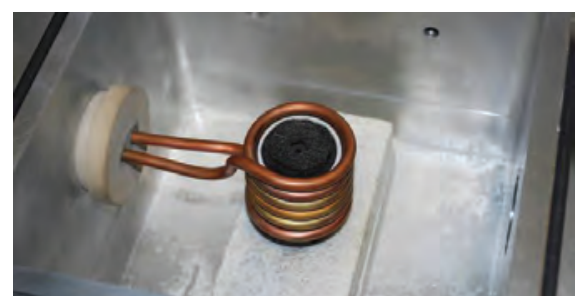

Fig. 11: Induction heated fixed-point device, view to inner components (induction coil with ceramic crucible, graphite fixed-point cell and graphite felt)

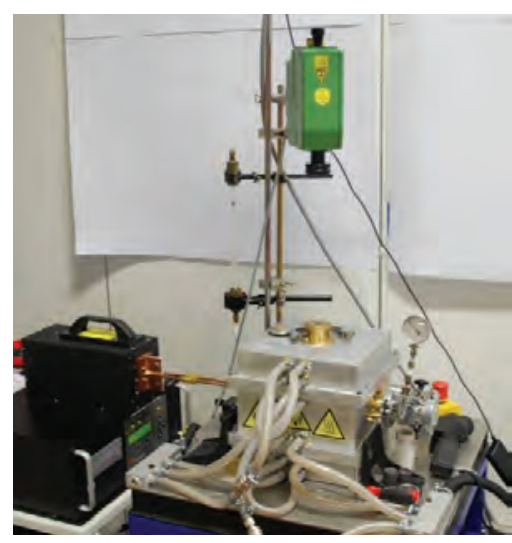

Fig. 12: Prototype of the induction heated fixed-point device at PTB Berlin, lab set-up for calibration of a pyrometer

The device has a water cooled and vacuumsealed housing with ports for connection to a vacuum pump, shielding gas supply and a special lead-through of the copper tubes of the induction coil. All equipment is installed in a 
19 inch rack for good system mobility. For safety reasons all important system parameters like shielding gas flow, water flow and temperature and residual oxygen content are measured and watched.

\section{Measurement results}

The induction heated fixed-point device was tested with $\mathrm{Cu}$ and $\mathrm{Fe}-\mathrm{C}$ fixed-point cells which have been built in the device successfully.

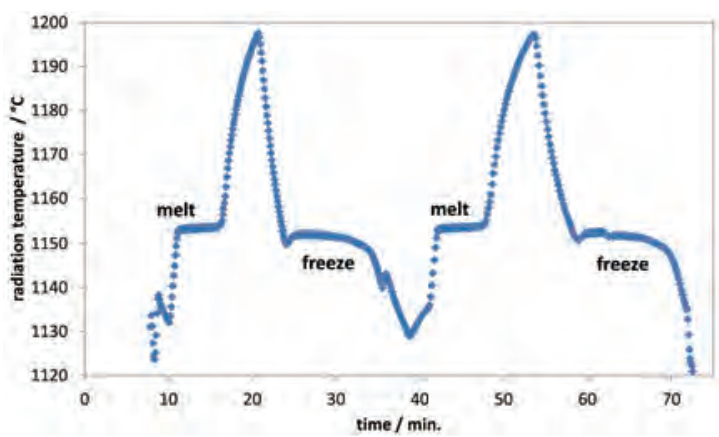

Fig. 13: Two melt and freeze cycles of a Fe-C fixedpoint cell heated with induction furnace, melting temperature $1153{ }^{\circ} \mathrm{C}$, pyrometer Infratherm IS12 (Impac Infrared $\mathrm{GmbH}$ ), wavelength $700-1100 \mathrm{~nm}$

The inflection points of the melt and freeze temperature curves agree with the theoretical values within a band of $\pm 1 \ldots 2$ Kelvin, i.e. within the measurement uncertainty of the used radiation thermometers.

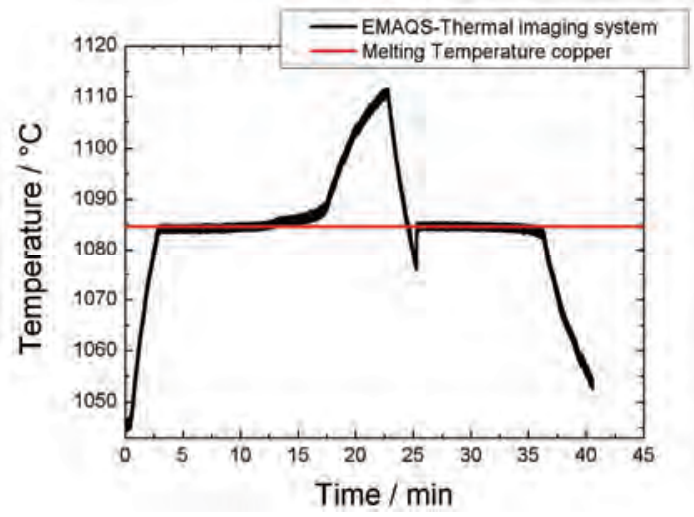

Fig. 14: Melt and freeze cycle of a Cu fixed-point cell heated with induction furnace, melting temperature $1084.6{ }^{\circ} \mathrm{C}$, measured with thermal imaging system E-MAqS (Fraunhofer IWS Dresden) after calibration with $\mathrm{Fe}-\mathrm{C}$ fixed point, measuring wavelength $740 \mathrm{~nm}$

\section{Conclusions}

The requirements for temperature measurement in laser heat treatment were investigated. Based on the results a new induction heated fixed-point device for calibration was developed. The prototype was constructed and manufactured by the PTB Berlin and tested with $\mathrm{Cu}$ and $\mathrm{Fe}-\mathrm{C}$ fixed-point cells for the calibration of temperature measurement devices, typically used in laser heat treatment, successfully. Further experimental investigations are planned to minimize the uncertainty of the new calibration method.

In the next stage of the project the emissivity of steel and cast iron in the temperature range between 1000 and $1500{ }^{\circ} \mathrm{C}$ is investigated and measured. The combination of precise and reproducible temperature measurement and emissivity correction will further reduce the uncertainty of the absolute temperature measurement with pyrometers and thermal imaging systems used for thermal processing.

The new possibilities for precise and reproducible temperature measurement in laser heat treatment processes are expected to improve the quality of industrial processes and open the door to new applications for difficult materials with strong dependence of the resulting material properties on the surface temperature in laser processing.

\section{Acknowledgements}

The work was undertaken in the content of the EURAMET joint research project "HiTeMS" (High temperature metrology for industrial applications).

The EMRP is jointly funded by the EMRP participating countries within EURAMET and the European Union.

Special thanks go to PTB Berlin (Germany) and NPL Teddington (UK) for training in high temperature fixed points and supply of calibration and measuring devices, pictures and measuring data.

\section{References}

[1] Bonss, S., Seifert, M., Brenner, B., Beyer, E., "Precision Hardening with High Power Diode Lasers using Beam Shaping Mirror Optics". Proceedings SPIE, 2003 San Diego, USA, 4973, p. 86-93

[2] Hollandt J et al., 2003/2007, "High-accuracy radiation thermometry at the National Metrology Institute of Germany, the PTB" High Temperatures - High Pressures 35/36(4) 379 415

[3] Anhalt, K., "Radiometric measurement of thermodynamic temperatures during the phase transformation of metal-carbon eutectic alloys for a new high-temperature scale above $1000^{\circ} \mathrm{C}$ ", dissertation, 2008, urn:nbn:de:kobv:83-opus19712, URL: http://opus.kobv.de/tuberlin/volltexte/2008/1971/

[4] EMRP A169 (REG), RESEARCHER GRANT CONTRACT NO. IND01-REG1, JRP: IND01 HITEMS http://projects.npl.co.uk/hitems/ 